\title{
CONFLICTOS POR EL AGUA EN GUANACASTE, COSTA RICA: RESPUESTAS AL DESARROLLO TURÍSTICO
}

\author{
WATER CONFLICTS IN GUANACASTE, COSTA RICA: \\ RESPONSES TO TOURISM DEVELOPMENT
}

Ernest Cañada

Recibido: 07/01/2019 - Aceptado: 08/04/2019

\begin{abstract}
Resumen
En este artículo se explora si los conflictos socioecológicos vinculados a procesos de desposesión por inversiones turístico-residenciales pueden ser analizados desde la perspectiva de la visión que construyen estos sectores de su relación con el turismo. Se analiza el caso concreto de Guanacaste, Costa Rica, donde se ha experimentado una gran intensidad tanto en el desarrollo de la inversión turística como en los conflictos ecológicos por el agua, entre las comunidades rurales y las inversiones en turismo residencial apoyadas por el Estado. La pregunta que surge es si en un contexto rural de estas características cristaliza un discurso de rechazo del turismo, como ha ocurrido en determinados destinos urbanos, o si, en el marco de intensos conflictos y negociaciones, los argumentos de los movimientos sociales comunitarios que reaccionan frente a estos mecanismos de desposesión entroncan con demandas de un turismo inclusivo.
\end{abstract}

Palabras clave: agua, conflicto socioecológico, Guanacaste, turismo, turismo inclusivo.

\begin{abstract}
In this article, we aim to examine whether socio-ecological conflicts linked to dispossession processes by residential tourism investments can be analyzed from the viewpoint that builds these sector's relationship with tourism. We analyze the specific case of Guanacaste, Costa Rica, where there has been high intensity both in the development of tourism investment and in ecological conflicts over water between rural communities and investments in residential tourism supported by the State. The question we ask ourselves is whether a discourse of rejection of tourism forms in a rural context of these characteristics, as has happened in certain urban destinations, or rather, in the framework of intense conflicts and negotiations, the arguments of the community social movements that react to these mechanisms of dispossession relate to the demands of an inclusive tourism.
\end{abstract}

Keywords: water, socio-ecological conflicts, Guanacaste, inclusive tourism, tourism. 


\section{Introducción}

En los últimos años, el crecimiento turístico en determinadas áreas del planeta, en especial en destinos urbanos, ha provocado que parte de sus habitantes percibieran masificación y saturación, lo cual ha tenido como consecuencia expresiones de males$\operatorname{tar} \mathrm{y}$, en ocasiones, de rechazo al turismo. Este fenómeno, que ha empezado a identificarse como overtourism, ha recibido una atención creciente por parte de la academia, autoridades públicas y medios de comunicación. La popularización del término empezó fundamentalmente a raíz del éxito editorial del libro de Elizabeth Becker, Overbooked: The Exploding Business of Travel and Tourism y de la repercusión en los medios de comunicación de algunas acciones de protesta contra los procesos de turistización de diversas ciudades europeas, en especial en Barcelona, Palma, Venecia y Florencia.

El fenómeno ha tenido como referencia destinos urbanos sometidos a una notable presión turística. La presencia masiva de turistas en un espacio concentrado y altamente densificado posibilita que parte de la población local identifique en el turismo la causa de múltiples malestares y problemas sociales: transformación de vecindarios al servicio del turismo, masificación y privatización de espacios públicos, incremento del costo de la vida, disminución de la oferta de vivienda para uso residencial, saturación del transporte público, aumento del ruido y pérdida de calidad de vida (Milano; Milano, Cheer y Novelli, 2018). Lo anterior puede dar lugar a ciertas manifestaciones de rechazo al turismo y a demandas de contención, incluso, de decrecimiento; estas van más allá de minorías activistas, de hecho, en algunas ciudades han alcanzado a sectores destacados de población en un creciente proceso de "politización desde abajo" (Colomb y Novy). La identificación de este creciente malestar ha dado pie a que organismos como la Organización Mundial del Turismo (UNWTO, por sus siglas en inglés) abrieran una discusión sobre la necesidad de diseñar políticas turísticas que ayuden a desactivar esta desafección creciente en destinos urbanos (UNWTO et al., 2018; UNWTO et al., 2019).

El concepto de overtourism adolece aún de un insuficiente desarrollo teórico, aunque ha motivado una creciente investigación en múltiples contextos alrededor del mundo, de manera que se enriquecen las perspectivas de análisis, como lo evidencia el libro recientemente editado por Milano, Cher y Novelli (2019). En este artículo usamos el término de overtourism para identificar la masificación turística asociada al malestar social. La percepción de masificación es necesariamente relativa. Es imposible fijar objetivamente una cifra de referencia para saber cuándo el turismo debe ser considerado demasiado. La idea de límite es una construcción social, subordinada a la opinión de la población implicada, por tanto, fruto de la participación ciudadana y la negociación, en situaciones forzosamente conflictivas (Saarinen, 2006). Por su parte, el malestar social al que hacemos referencia puede traducirse en formas diversas, las cuales pueden ir desde el conflicto social abierto hasta formas de resistencia menos evidentes (Scoot). 
El debate actual sobre el fenómeno overtourism puede ayudar a ampliar la preocupación y el análisis de porqué y cómo en determinados contextos el crecimiento turístico ha dado lugar a crecientes manifestaciones de malestar entre la población local como respuesta a lógicas excluyentes del desarrollo turístico por parte del capital. En el ámbito rural esta perspectiva ha sido menos analizada, pero puede ser un marco de interés para poder interpretar ciertas reacciones de grupos comunitarios ante el desarrollo turístico.

\section{Objetivos y metodología}

El objetivo de este artículo es explorar si ciertos conflictos socioecológicos vinculados a procesos de desposesión por inversiones turístico-residenciales, como los que se han producido en Guanacaste, Costa Rica, pueden ser mejor interpretados al incorporar la visión que construyen estos sectores de su relación con el turismo. De forma más concreta, se analiza qué tipo de discursos se cristalizan en los movimientos sociales de base comunitaria, en un contexto rural, ante lo que podríamos identificar como overtourism, en tanto reacción de malestar de la población local ante procesos de desarrollo turístico.

Ahora bien, este texto se incluye en un proceso de investigación en curso más amplio sobre las implicaciones del desarrollo turístico en Centroamérica, desde la perspectiva de la tensión existente entre procesos de exclusión e inclusión y con un enfoque basado en los derechos humanos. El artículo tiene carácter de reflexión teórica a partir de la revisión bibliográfica, pero también de la experiencia personal de trabajo de campo en Guanacaste. Dado su carácter exploratorio y de reflexión, se ha decidido no usar los resultados específicos de la investigación de campo, con el fin de priorizar la presentación de nuevas perspectivas que puedan ayudar a plantear nuevas preguntas y líneas de investigación. La presencia en la zona de estudio empieza a partir de una relación de colaboración con el grupo ecologista Confraternidad Guanacasteca, iniciada en 2008, y con diferentes organizaciones comunitarias de Guanacaste. Desde entonces, se han realizado visitas periódicas para documentar distintos conflictos socioambientales vinculados al turismo; las dos últimas se realizaron en enero de 2017 y en septiembre de 2018, para desarrollar trabajo de campo en diversas comunidades rurales de Guanacaste, en especial Sardinal, con el fin de analizar los conflictos socioecológicos que vinculan el agua con el desarrollo turístico-residencial en Guanacaste (Cañada, 2019).

\section{¿Overtourism en el mundo rural? Elementos en juego}

En el mundo rural la percepción de saturación turística adquiere un aspecto dual: por la aglomeración de turistas en ciertos territorios - playas, espacios urbanizados o ciertas áreas de montaña, por ejemplo- y por la sustracción de recursos 
naturales para posibilitar el desarrollo turístico. Así, en espacios rurales-costeros de Centroamérica se ha identificado que la presión del capital turístico, factor fundamental de transformación territorial (Bianchi, 2002; Bianchi, 2011), sobre los medios de vida de la población rural -la tierra, el agua o ecosistemas costeros como manglares, o bien, la reorganización espacial que implica este desarrollo turístico- ha acrecentado los conflictos socioambientales (Cañada, 2018; Cañada y Gascón), en el marco de una conflictividad en ascenso asociada al crecimiento turístico en la región (Cañada, 2010). Esta dinámica, a su vez, profundiza las lógicas de dependencia a través del turismo descrito en otros contextos de países periféricos (Britton, 1982; Britton, 1996) y de desposesión (Harvey, 2003).

Para su reproducción, el capital turístico e inmobiliario reorganiza el espacio rural con áreas en las que concentra la oferta de atención directa al turista y otras, mucho más extensas, en las cuales se instalan las infraestructuras necesarias para facilitar ese desarrollo turístico: carreteras, generación de energía y su red de distribución, pozos de agua y acueductos, entre otros, lo cual pone en riesgo la reproducción de las comunidades rurales, a causa del despojo de medios de vida, como la tierra, el agua o ecosistemas marítimos (Cañada, 2018). Esta dinámica tiende a desestructurar a las comunidades rurales preexistentes, mucho más allá de los territorios en los cuales se produce propiamente la actividad turística. La creciente conflictividad rural, motivada a diferentes escalas por el turismo, es así interpretada como una manifestación de overtourism en el mundo rural (Milano, 2018).

La pregunta que en consecuencia nos planteamos es si, en un contexto rural de estas características, se cristalizan discursos de rechazo al turismo o movimientos sociales parecidos a los que se han llegado a producir en ciertos destinos urbanos, descritos en diversos estudios (Colom y Novy, 2016; Milano y Mansilla); o si, más bien, en el marco de intensos conflictos y negociaciones, los argumentos de los movimientos sociales comunitarios que reaccionan en la ruralidad frente a los impactos del desarrollo turístico-residencial entroncan con demandas de un turismo inclusivo, entendido como un desarrollo turístico en el cual los sectores empobrecidos o marginados de la economía dominante ven ampliadas sus posibilidades de acceso al turismo, como consumidores y como productores, y en el que, además, tienen una mayor participación en la toma de decisiones sobre las actividades turísticas y la distribución de los beneficios generados (Scheyvens y Biddulph).

Este texto analiza el caso de la provincia de Guanacaste en Costa Rica. La zona ha experimentado una elevada intensidad tanto en el desarrollo de la inversión turístico-residencial (Van Noorloos, 2012; Román, 2007; Román, 2009) como en los conflictos socioecológicos vinculados con el agua entre comunidades rurales e inversiones turístico-residenciales apoyadas por el Estado (Hernández y Picón, 2011). Lo anterior ha dado lugar a una creciente contradicción entre las políticas neoliberales impulsadas 
por el Estado, las cuales promovieron este desarrollo turístico-residencial, a través de políticas de conservación ambiental de las que ha hecho bandera el país, y la supervivencia de comunidades rurales en espacios revalorizados por el capital turístico e inmobiliario (Barrantes Reynolds).

El acceso al agua se ha convertido en un factor limitante para el desarrollo turístico-residencial, por tanto, estratégico para sus capitales (Cole; Cole y Browne; Hof y Blázquez-Salom). Cuando la competencia por el agua, entre las necesidades básicas de la población local y los intereses de las inversiones turístico-residenciales, entra en contradicción, a causa de su escasez, surgen intensos conflictos socioambientales o ecológico-distributivos. Entendemos estos conflictos como disputas distributivas vinculadas con la asignación de unos beneficios y unos costos (socioecológicos) perceptibles por parte de los actores que se movilizan con la intención de incidir en esta distribución (MartínezAlier). Este proceso no sería posible sin transformar en mercancía y privatizar bienes comunales como el agua, en el marco de procesos de acumulación por desposesión, entendida como el uso de métodos de acumulación originaria para sostener de forma permanente el sistema capitalista (Harvey, 2003; Harvey, 2014), con los que el capital turístico hegemoniza determinados territorios a través de políticas de carácter neoliberal (Bakker, 2007; Bakker, 2010).

\section{Desarrollo turístico en Guanacaste}

Desde finales de los años noventa, la provincia de Guanacaste, al noroeste de Costa Rica, ha experimentado un fuerte desarrollo turístico-residencial. La fiebre constructora, y en parte especulativa, que se vivió durante los años 2000, hasta el inicio de la crisis financiera internacional en 2008, posicionó a la provincia como el principal destino turístico de Centroamérica (Cañada, 2013; Román, 2009). Tras una fuerte recesión, a partir de 2013 se ha experimentado una ligera recuperación de la actividad turístico-residencial que, sin embargo, se ha visto frenada por una demanda internacional que no muestra la misma capacidad de compra que en los años previos a la crisis y por la falta de suficiente disponibilidad de agua potable para los desarrollos turístico-residenciales, según apreciaciones de diferentes funcionarios del Instituto Costarricense de Acueductos y Alcantarillados (AyA) consultados. Esta escasez de agua en las zonas litorales, como Playa Ocotal o Playas del Coco, que son las de mayor atractivo turístico, ha provocado una presión recurrente por parte del sector turístico para que el Estado hiciera obras de infraestructura que les garantizara su acceso, a su vez, ha sido el origen de numerosos conflictos socioambientales, como los que se han producido en Sardinal de Carrillo (Fernández, 2009; Navas; Navas y Cuvi; Silva, 2016; Silva, 2019), Nimboyores (Blanco, 2017; Kuzdas; Silva, 2018) o Playa Potrero (Bolaños). 
Figura 1

MAPA DE GUANACASTE

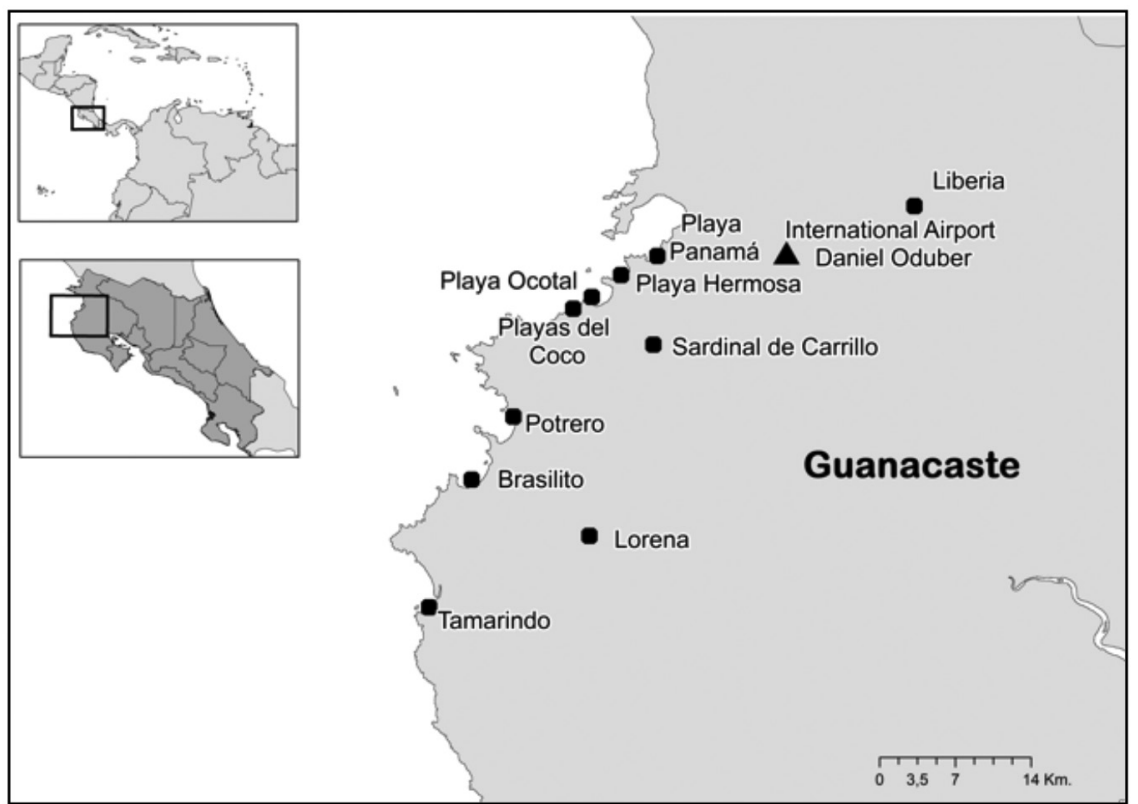

Fuente: Elaboración propia.

Históricamente, la provincia de Guanacaste estuvo vinculada a la ganadería, con una presencia destacada de ciudadanos estadounidenses que compraron tierras (Edelman, 1998), y a la agricultura para exportación, principalmente de caña de azúcar y arroz. A raíz de la decadencia de las actividades ganaderas en los años ochenta del siglo XX (Cabrera), como consecuencia de la caída del precio de la carne en el mercado internacional y las propias presiones de Estados Unidos para que su producción disminuyera, autoridades de la provincia y empresarios locales visualizaron en el turismo una alternativa que, además, podía beneficiarse de los vínculos económicos que durante décadas se habían construido con la inversión norteamericana (Blanco, 2018; Van Noorloos, 2012).

Por supuesto, el capital internacional estaba ávido de encontrar nuevos destinos en los cuales expandirse y poder reproducirse, con lo cual se daría respuesta a las crisis recurrentes del capitalismo, en lo que se ha denominado soluciones espacio-temporales (Harvey, 2014). Así, Costa Rica, en especial Guanacaste, permitió dar continuidad a procesos de expansión turística previamente vividos en México y diversas áreas del Caribe, como República Dominicana y Cuba (Blàzquez et al., 2011; Cañada, 2013). La incorporación de Costa Rica en estos mercados turísticos internacionales coincidió, durante la primera década de los años 2000, con el desarrollo de un creciente mercado 
global de turismo residencial (Janoschka, 2011), lo cual explica, por las características de este tipo de productos, la intensidad con la que se produjo su desarrollo.

A su vez, el gobierno de Costa Rica, en el marco de las políticas de orientación neoliberal que buscaban un nuevo encaje en la economía global, otorgó amplias facilidades para promover la inversión y el desarrollo turístico en el país, en particular en la provincia de Guanacaste. Lo anterior dio lugar a incentivos fiscales, regulados por ley desde 1985 y modificados en 2010, de manera que las empresas que cumplen con la ley podrían beneficiarse de la exención de impuestos en la compra de bienes utilizados para su operación o el suministro de combustible a bajo precio, entre otras cosas; concesiones de importantes áreas de territorio en la zona marítimo terrestre; construcción de infraestructuras clave, por ejemplo el Aeropuerto Internacional Daniel Oduber en Liberia, el cual permitiría vuelos chárter a Norteamérica; y apoyo directo a la instalación de megaproyectos turísticos (Barrantes Reynolds; Janoschka, 2011; Van Noorloos, 2012).

También se ha destacado que las autoridades del país otorgaron mucha libertad a inversionistas y desarrolladores en la forma de interpretar las regulaciones ambientales y espaciales. Además, el control sobre su aplicación fue muy deficiente, tanto por capacidades de personal como financieras, y por la propia decisión política de facilitar esas inversiones con una lectura laxa de las evaluaciones de impacto ambiental o en la forma en que se llevaron a cabo distintos planes reguladores, en muchos casos adaptados a los intereses de los mismos inversionistas privados (Román, 2007).

En la práctica esto supuso una verdadera desregulación que facilitó el crecimiento desordenado de ese desarrollo turístico-residencial (Van Noorloos, 2012), lo cual afectó en especial a la Zona Marítimo Terrestre, donde se produjeron numerosas irregularidades (Román, 2009). Esto dio lugar a crecientes tensiones entre la ejecución de la política neoliberal, la cual potenciaba la atracción de inversión extranjera, y el desarrollo de una política ambiental de conservación, que se saldó claramente a favor de los negocios turístico-residenciales (Barrantes Reynolds), como ha sucedido en otros contextos (Bianchi, 2018).

El municipio de Jacó, en la provincia de Puntarenas, brinda un ejemplo extremo de degradación ambiental y exacerbación de las desigualdades sociales a través del desarrollo turístico-inmobiliario con fragmentación jurisdiccional, debilidades regulatorias, coordinación deficiente, actuación de la autoridad pública lenta y planificación y desarrollo incoherentes (Irazábal).

Por su parte, Guanacaste se benefició de la proximidad con Nicaragua, de donde se desplazaron numerosas personas inmigrantes para trabajar en la construcción, muchas de ellas en situación de ilegalidad, de manera que los desarrolladores pudieron pagarles salarios más bajos que a la población costarricense (Acuña; Morales), incluso, no garantizarles condiciones de vida adecuados en alojamiento y servicios básicos (Navarro, 2013). Este acceso a mano de obra inmigrante en condiciones de vulnerabilidad supuso un importante ahorro tanto para los inversionistas como para el propio Estado costarricense. 
Este desarrollo turístico adquirió diversas formas. Al turismo tradicional se le añadió un activo mercado de turismo residencial vinculado a una migración por estilo de vida (Van Noorloos, 2013; Van Noorloos, 2016). De este modo, además de hoteles de diversas categorías, incluida la presencia de grandes cadenas internacionales, norteamericanas y españolas, sobretodo, se produjo una oferta variada de urbanizaciones y condominios, además de campos de golf y puertos deportivos. A través de una activa política de concesiones, el Estado costarricense trató de atraer a grandes inversionistas que, entre otros asuntos, impulsaron iniciativas como el Polo Turístico del Golfo de Papagayo, declarada de utilidad pública para uso turístico en 1979 y con una ley específica para su regulación desde 1982 (Hernández y Picón, 2011). A estos distintos proyectos se le sumaron iniciativas particulares de construcción de casas en parcelas individuales o pequeñas urbanizaciones (Janoschka, 2011). De este modo, la dinámica inmobiliaria llegó a transformar las formas de ocupación de la zona costera, incluso, de otras áreas que juegan un rol clave en la provisión de servicios públicos y privados para esta (Román, 2009).

El fuerte desarrollo turístico residencial que se produjo en Guanacaste, en particular durante los años 2000 hasta el inicio de la crisis financiera internacional en 2008, no podría explicarse sin la enorme inversión extranjera, en especial de Estados Unidos, que llegó a la provincia. En el 2007, la inversión extranjera directa en Costa Rica vinculada a turismo y sector inmobiliario, según datos del Banco Central de Costa Rica, alcanzó algo más de 966 millones de dólares, un 51 por ciento del total que llegó al país. La inversión inmobiliaria entre 2002 y 2007 fue especialmente aguda, solo superada por el sector industrial, como puede verse en la Tabla 1.

Tabla 1

INVERSIÓN EXTRANJERA EN BIENES RAÍCES POR REGIÓN DE ORIGEN DEL CAPITAL, 2004-2007. (EN MILLONES DE DÓLARES)

\begin{tabular}{lccccc}
\hline & 2004 & 2005 & 2006 & 2007 & $2004-2007$ \\
\hline Estados Unidos & 103.95 & 71.10 & 224.03 & 351.86 & 750.9 \\
Resto de América del Norte & 7.47 & 23.44 & 31.04 & 42.26 & 104.2 \\
Centroamérica & 1.02 & 2.26 & 2.88 & 15.19 & 21.4 \\
América del Sur & 6.11 & 3.87 & 8.52 & 23.94 & 42.4 \\
Europa & 39.01 & 58.32 & 68.50 & 161.13 & 327.0 \\
Asia & 5.62 & 2.20 & 11.77 & 19.47 & 39.06 \\
Otros & 13.39 & 63.34 & 17.23 & 17.41 & 111.37 \\
\hline Total & 176.56 & 224.54 & 363.97 & 631.27 & 1396.3 \\
\hline
\end{tabular}

Fuente: Román, 2009. 
Se ha destacado también que, durante esos años, Costa Rica tuvo el mayor crecimiento en volumen y precios del sector inmobiliario en el mundo (Gordon et al., 2010).

En términos provinciales, Guanacaste fue la provincia en la que se concentró la mayor cantidad de inversión extranjera en el sector inmobiliario (Barrantes Reynolds). Se calcula que en menos de una década Guanacaste concentró cerca de mil millones de dólares en inversiones inmobiliarias (Janoschka, 2009). Una parte muy destacada de la construcción de vivienda nueva fue para satisfacer la demanda turístico-residencial, no las necesidades de la población local (Román, 2009). El origen de esta inversión en el sector inmobiliario fue fundamentalmente de Estados Unidos, la cual en 2007 alcanzó el 55.7 por ciento de la inversión extranjera; y, en menor medida, de otros países: Canadá, Alemania, España, Holanda, Italia (Román, 2009).

Se ha destacado, también, la importancia del capital costarricense, en muchos casos asociado a inversiones de origen norteamericano (Van Noorloos, 2012). Entre los desarrolladores destacan grandes empresas internacionales de inversión inmobiliaria a largo plazo; empresas inmobiliarias con trayectoria en el sector de la construcción (casas, centros comerciales, condominios u hoteles) y que se orientaban a inversionistas extranjeros también con perspectivas a largo plazo; $y$, finalmente, oportunistas de diversas nacionalidades, interesados en la compraventa a corto plazo, con la esperanza de conseguir un beneficio inmediato, con un comportamiento claramente especulativo (Román, 2009). El perfil de los compradores finales fue también diverso: extranjeros con alto poder adquisitivo acostumbrados a invertir en viviendas para uso vacacional en diversas partes del mundo; baby-boomers de EE. UU. y Canadá que buscan oportunidades para mejorar su nivel de vida una vez retirados; inversionistas costarricenses con altos ingresos.

La evolución del desarrollo turístico en Guanacaste se vio muy influenciada por la crisis financiera internacional iniciada en 2008. La construcción en la zona fue en ascenso durante todos los años 2000 hasta una importante caída entre 2008 y 2012. A partir de ahí, se ha producido una muy ligera recuperación, que en ningún caso ha vuelto a los niveles previos a la crisis. 
Figura 2

EVOLUCIÓN DE LA CONSTRUCCIÓN EN GUANACASTE, SEGÚN ÁREA CONSTRUIDA EN M²

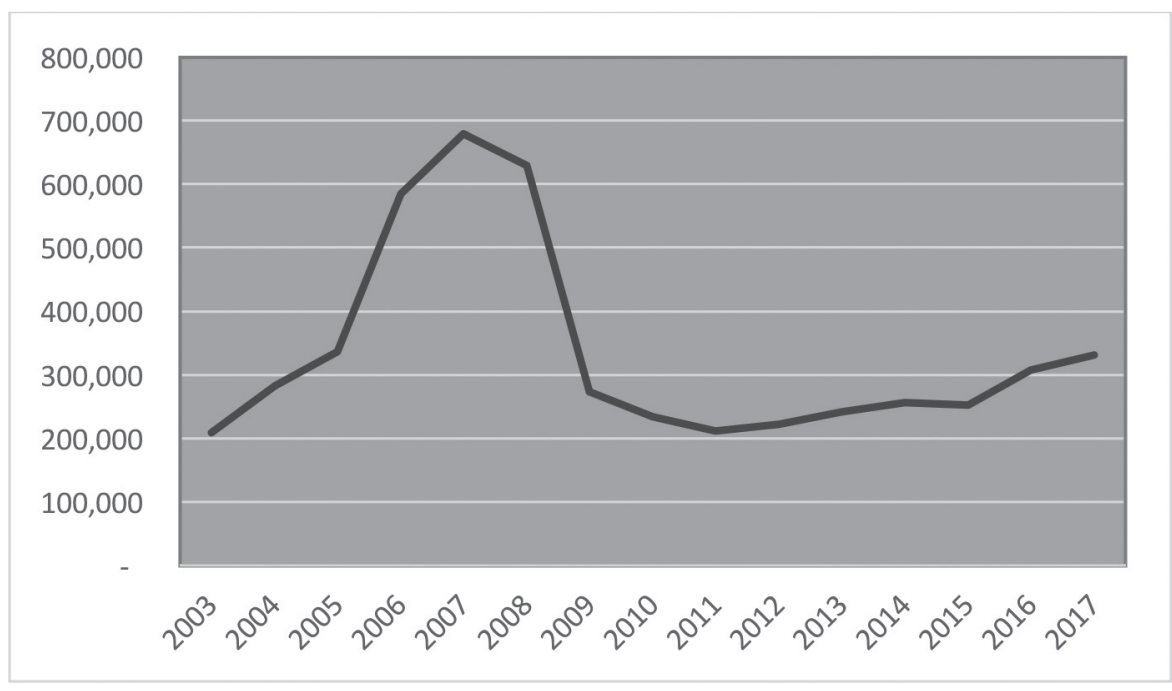

Fuente: Programa Estado de la Nación con datos de INEC, Base de datos de área construida.

En este desarrollo la dinámica especulativa provocó un fuerte incremento de precios. Entre el 2000 y el 2011, el precio de las tierras aumentó un 17.7 por ciento anualmente y en las casas y apartamentos un 24.3 por ciento (Van Noorloos, 2012). En algunos lugares, como Tamarindo, estos incrementos pudieron ser mucho mayores (Pera). Esto implicaba que no todas las inversiones supusieron el inicio de nuevas actividades económicas, más allá de la compra y venta de terrenos específicos, o, como mucho, la provisión de nuevos servicios para facilitar su venta.

La transformación económica que supuso este declive de las actividades agropecuarias tradicionales y el ascenso del turismo residencial se tradujo también en términos de ocupación. Entre 2001 y 2011, la distribución de la población ocupada por ramas de actividad en la Región Chorotega, la cual corresponde a la totalidad de la provincia de Guanacaste y al cantón alajuelense de Upala, muestra una caída del sector agropecuario del $25.3 \%$ al $13.6 \%$ y un incremento de la participación del trabajo en hoteles y restaurantes del $9.4 \%$ al $12.8 \%$. Significativamente, el sector de actividad que más creció en ese período fue el de servicio doméstico, el cual pasó de un $3.5 \%$ a un $10 \%$ (Estado de la Nación). La participación de la construcción se mantuvo prácticamente estable, pasó de un $6.4 \%$ a un $7 \%$, aunque se supone un elevado subregistro debido a la importancia de la mano de obra de origen nicaragüense en situación de ilegalidad, por tanto, no registrada. 
En términos de permisos de construcción, según datos proporcionados por el Programa Estado de la Nación, a partir de la base de datos del Instituto Nacional de Estadística y Censos de Costa Rica (INEC), la provincia alcanzó un máximo de 4568 en el 2008, el cual cayó hasta el mínimo de 2657 en 2012; a partir de ahí, empezó a crecer de forma ligera hasta alcanzar los 3073 en 2016, para bajar de nuevo a 2836 en 2017, lo cual pone en evidencia las incertidumbres existentes en torno a la posibilidad de una reactivación del modelo de desarrollo previo a la crisis (Estado de la Nación).

Dos factores pueden ayudar a explicar las limitaciones de este proceso de recuperación. Por una parte, la demanda internacional del tipo de productos inmobiliarios orientados hacia una migración por estilo de vida de poder adquisitivo medio-alto de origen norteamericano que se ha impulsado en Guanacaste parece está lejos del dinamismo que mostró en los años previos a la crisis. Por su parte, desde una perspectiva local, inversionistas y autoridades sostienen que la imposibilidad de garantizar agua potable en los desarrollos costeros estaría limitando la capacidad de poner en marcha proyectos ya construidos o atraer nuevas inversiones.

\section{Turismo y conflictos por la sobrexplotación del agua en Guanacaste}

La provincia de Guanacaste está sujeta a un clima tropical seco y con una pluviosidad poco abundante durante parte del año, a lo que se han sumado los efectos del cambio climático, con sequías más agudas en los últimos años (Picón et al., 2004). Así la disponibilidad de agua potable en las zonas del litoral se ha convertido en un factor crítico para el éxito o fracaso de muchos proyectos turístico-residenciales (Hernández y Picón, 2013; Picón et al., 2014).

Para las autoridades públicas, inversionistas, propietarios de tierra y empresarios vinculados a actividades de servicio al turismo, ampliar la capacidad de abastecimiento de agua en las zonas costeras ha sido una de sus prioridades. Según las autoridades del Instituto Costarricense de Turismo (ICT), los problemas del agua en la provincia no tienen que ver con la sobreexplotación turística del territorio, sino que son resultado de una suma de factores combinados, tal como expresa su director de Planeamiento y Desarrollo:

Desde luego, la suma de impactos no es una consecuencia directa del volumen de ocupación sino más bien de la ausencia de prácticas adecuadas de manejo, tales como los sistemas de tratamiento de aguas servidas, la protección de cuerpos de agua, la disposición de desechos, etcétera. Igualmente, la problemática no es atribuible a una sola fuente sino más bien a un conjunto de impactos que genera todo el espectro de ocupación, incluyendo las residencias, comercios, negocios, agricultura, ganadería, pequeña industria, pesquería y otros (Lizano 12).

Por medio de la construcción de pozos, en un primer momento, y de acueductos, después, se trató de resolver un problema acuciante para un modelo de desarrollo 
basado en la explotación turístico-residencial del territorio. Sin embargo, la sobreexplotación de algunos acuíferos en la zona litoral, sin una adecuada planificación y falta de control por parte de las autoridades públicas de las irregularidades cometidas (Honey et al.), provocó que disminuyera la disponibilidad de agua en algunos acuíferos; que quedaran totalmente desabastecidos, como ocurrió en diversos pozos del municipio de Tamarindo, con una intensa saturación turística y descontrol socioambiental (Picón y Baltodano, 2006; Pera; Román, 2006); o bien, que llegaran a salinizarse, como ocurrió en el acuífero de Playa Panamá, en el cantón de Carrillo (Cañada, 2016; Cruz; Quirós). Al extraer agua en grandes cantidades, baja el nivel piezométrico, entonces, el agua de mar puede introducirse y contaminar el acuífero, lo que se conoce como intrusión marina. Estos casos de salinización dieron pie a que las autoridades retomaran con más fuerza la idea de la construcción de acueductos desde acuíferos situados al interior, de manera que se pueda suministrar agua a las costas, como ha sido el caso de Trancas para abastecer la Bahía de Papagayo y el de Sardinal con Playas del Coco y Playa Ocotal.

En este contexto, desde finales de los años noventa, la provincia de Guanacaste ha vivido con intensidad una sucesión de conflictos entre comunidades y proyectos vinculados a intereses hoteleros y residenciales en los que la problemática del agua ha tenido un especial protagonismo. En estos, la participación de las mujeres ha sido muy activa, en parte por el estrecho vínculo cultural construido entre ellas y el uso y manejo del agua en un contexto marcado por desigualdades de género (Cole y Ferguson, 2015). Estos conflictos socioambientales han estado ampliamente documentados y caracterizados (Hernández y Picón, 2011; Ramírez, 2008), en especial por su vinculación con la gestión del agua (Alpízar), y se inscriben en un ciclo de conflictividad causada por el turismo que se ha agudizado en Centroamérica y en particular en Guanacaste (Cañada, 2010; Cañada, 2013).

Los principales conflictos vinculados al uso del agua entre comunidades rurales y el desarrollo turístico-residencial en Guanacaste han estado motivados por la extracción de agua de los acuíferos y su canalización para dar servicio a iniciativas turísticas, como los casos de Sardinal (Fernández, 2009; Navas; Navas y Cuvi; Silva, 2016; Silva, 2019) y Nimboyores (Blanco, 2017; Kuzdas; Silva, 2018), así como por cambios en las formas de gestión del agua en los cuales el Estado ha querido tomar el control de estructuras de gestión comunitarias, con el fin de garantizar el servicio a proyectos turísticos, como el caso de Playa Potrero (Bolaños). Las comunidades se abastecen de los acuíferos principalmente para su consumo cotidiano y para actividades de riego.

Los desarrollos turísticos-residenciales han generado una presión sobre este recurso, lo cual ha sido visto como un riesgo que amenaza sus medios de vida, de ahí su resistencia ante proyectos de infraestructura o pérdida de control sobre su gestión. El caso de la salinización del acuífero de Playa Panamá tuvo un efecto contradictorio. Por una parte, se convirtió en una señal de alarma para los pobladores de muchas 
otras comunidades rurales debido al riesgo que suponía la sobreexplotación turística; pero, por otra parte, también fue leído por las autoridades como un argumento a favor de la necesidad de extraer agua de acuíferos del interior y poder garantizar el abastecimiento del litoral, no solo para desarrollos turísticos, sino también para el conjunto de la población, según declaraciones de funcionarios del AyA consultados personalmente (Cañada, 2019).

Otro aspecto crítico para las comunidades rurales ha sido la contaminación provocada por el vertido irregular de aguas residuales por parte de desarrollos hoteleros y residenciales al mar, ríos, vertederos, incluso, en carreteras secundarias, donde por infiltración ha podido contaminar también algunos acuíferos. Algunos casos, como el del Hotel Occidental Allegro Papagayo, en Playa Hermosa, en 2008, provocaron un escándalo público por el vertido ilegal de aguas negras, lo cual hizo eco en los medios de comunicación costarricenses, de manera que se obligó a las autoridades sanitarias a clausurar el hotel hasta que se construyeran las plantas de tratamiento requeridas por su capacidad de alojamiento (Ávalos; Retana).

En contraste, no todos los problemas de desabastecimiento o salinización de los acuíferos han derivado en conflictos socioambientales entre comunidades y desarrollos turístico-residenciales. En el caso de Tamarindo, por ejemplo, iniciado en los años noventa como destino especializado en surf, durante los años 2000, vivió un intenso desarrollo turístico que dio lugar a este tipo de afectaciones en los acuíferos de los que se abastecía; además de otros impactos negativos como desplazamientos de población, alza de precios, masificación, incremento de la inseguridad asociada a la venta de droga, asaltos y prostitución, no se han producido conflictos por el agua de la naturaleza e intensidad de los anteriormente mencionados (Silva y Morera, 2018).

De forma exploratoria, a partir de entrevistas tanto del casco urbano como de comunidades aledañas, se constata que entre la población local no se percibe una identificación del turismo como causante de estos problemas. Las reacciones ante problemas con el agua no se plantean en términos de oposición a las formas que adquiere el desarrollo turístico. El malestar de una parte de la población más bien se focaliza en problemas de seguridad vinculados a ciertos colectivos de población inmigrante a quien se identifica con la realización de actividades delincuenciales, en especial colombianos, venezolanos y nicaragüenses. Así, el turismo se sigue viendo como la principal fuente de ingresos y oportunidades de la zona. De todos modos, en posteriores investigaciones sería necesario profundizar en las razones por las cuales los fenómenos de la contaminación y el desabastecimiento de las fuentes de agua se cristalizan en conflictos socioambientales en determinadas comunidades, pero en otras no.

Ahora bien, en distintos lugares de la provincia, especialmente en los núcleos de población más cercanos al litoral, se han articulado diferentes asociaciones de desarrollo formadas por población local, las cuales han demandado la construcción de 
infraestructura que permitiera el abastecimiento de agua para los desarrollos turísticoresidenciales. Identifican en el turismo la principal fuente de empleo y oportunidades para la venta de propiedades y la dinamización económica de negocios orientados al turismo. En la demanda de estas infraestructuras también han jugado un papel activo los residentes extranjeros, como en el caso de Playa Hermosa, y que, si bien no llegaron a conformarse como una asociación formal, sí han actuado como grupo de presión ante las autoridades locales, especialmente sobre las municipalidades, el ICT y el AyA (Cañada, 2019).

El caso más reciente es el de Playa Marbella, una comunidad de unos cuatrocientos habitantes a poco más de 40 kilómetros del municipio de Santa Cruz, Guanacaste, donde un desarrollador estadounidense, quien es dueño y vende lotes para grandes desarrollos turísticos, es a su vez gestor de la Asociación Administradora de Sistemas de Acueductos y Alcantarillados Comunales (ASADA) Posada del Sol, la cual gestiona los pozos de agua de los cuales se abastecen estos desarrollos (Sancho).

De este modo, los grupos comunitarios que han sostenido conflictos con desarrollos turístico-residenciales por el agua, de forma creciente se han visto confrontados por otros colectivos de población que defendían posiciones contrarias.

\section{Resultados: Contención, atención e integración en otro modelo turístico}

Para muchas comunidades, el crecimiento del turismo se ha identificado como una amenaza para sus medios de vida. Los sectores comunitarios más activos, que se han movilizado en los últimos años en conflictos vinculados con el agua, han formulado una visión crítica del desarrollo turístico que pone en duda la lógica de las bondades de este tipo de actividades. La percepción de desplazamiento y sustracción de recursos básicos sumado a los pocos beneficios que ha comportado el turismo está claramente presente. Los términos en los cuales se ha planteado el debate por parte de estos sectores se construye en torno a tres grandes argumentos: 1) se cuestiona la lógica de la necesidad del crecimiento turístico permanente y se defiende cierta contención; 2) se demanda mayor atención a las necesidades de los sectores más vulnerables, cuyos intereses se consideran descuidados por las autoridades en contraste con las demandas de los desarrollos turístico-residenciales; y 3) se aspira a una mayor integración en los beneficios de un desarrollo turístico más inclusivo. Sin embargo, no se identifican expresiones de rechazo al turismo, ni siquiera en los sectores más movilizados, sino en las lógicas desiguales y de desposesión con las que se ha impuesto su desarrollo.

La idea de la necesidad de limitar de uno u otro modo la expansión del turismo ha estado presente desde hace tiempo, en especial durante los años 2000, cuando el crecimiento turístico e inmobiliario fue más intenso. En este ambiente, Monseñor Vitorino Girardi Stellin, obispo de la diócesis de Tilarán-Liberia, pudo formular la propuesta de una moratoria a nuevas concesiones de aguas con fines de explotación turística y comercial en una carta pastoral fechada el 19 de junio de 2009 donde afirmaba: 
Por todo lo anterior, con varias organizaciones guanacastecas creemos conveniente proclamar de inmediato una moratoria definitiva a la concesión de aguas con fines de explotación turística y agroindustrial hasta tanto no exista una planificación del desarrollo que efectivamente responda a criterios de sostenibilidad y que tome en cuenta de forma corresponsable y participativa a las comunidades involucradas (Girardi Stellin 30).

Sin embargo, la crisis económica internacional a partir de 2008, la cual conllevó un retraimiento en la inversión, coincidió con la paralización de obras como el acueducto de Sardinal, gracias a la movilización comunitaria. Lo anterior ha significado que los sectores empresariales y las asociaciones de desarrollo hayan podido atribuir la falta de empleo y oportunidades en el área a las limitaciones impuestas al turismo, y el discurso sobre este punto de vista puede haber permeado a sectores de la población. En consecuencia, en los últimos años los argumentos a favor de la contención turística del movimiento comunitario encontraran menos eco entre el resto de la población, según se desprende de entrevistas a diferentes personas vinculadas a asociaciones comunitarias de Sardinal y Playa Potrero (Cañada, 2019).

A su vez, los movimientos comunitarios plantean otro argumento en torno a cierta sensación de agravio comparativo por el contraste entre la preocupación y atención de las autoridades públicas a las demandas de los desarrollos turístico-residenciales, frente a distintos problemas no resueltos de los sectores más vulnerables, vinculados también con el agua. Esta insatisfacción se plasmó de forma explícita en el proceso de diálogo entre el gobierno de Costa Rica y el Comité pro-Defensa del Agua de Sardinal y la organización ecologista Confraternidad Guanacasteca por la construcción del acueducto de Sardinal a Playas del Coco y Playa Ocotal entre los meses de enero y abril de 2018. El sector comunitario cuestionó la necesidad de estas obras, que identificaba fundamentalmente con las necesidades de los proyectos turístico-residenciales, mientras no se resolvían problemas como la falta de acceso al agua potable en algunos barrios y comunidades rurales de la zona. Según las autoridades gubernamentales, esto era debido a que buena parte de estos pobladores no cumplían todos los requisitos legales en sus casas -como no tener títulos de propiedad o no estar escriturados debidamente a su nombre- y, por tanto, no podían suministrarles agua potable.

Para los comunitarios, el derecho al acceso al agua potable debería estar por encima de cualquier otro argumento. Igualmente cuestionaron que no se acabaran de resolver los problemas de comunidades que reciben aguas contaminadas con arsénico (Cañada, 2019). Este malestar se amplifica con la percepción de impunidad con la que actúan algunos desarrolladores que han cometido irregularidades sin mayores consecuencias, lo cual acrecienta la desconfianza hacia las autoridades públicas, que en contextos de tensión creciente en estos conflictos son acusadas de corrupción.

Finalmente, el tercer gran argumento de los movimientos comunitarios movilizados en la defensa del agua se focaliza en el cuestionamiento de los beneficios reales 
de este desarrollo turístico-residencial y la demanda de una mayor integración de la población local en sus beneficios. En las comunidades del interior se cuestiona que los efectos positivos del desarrollo turístico, en términos de dinamización económica por empleo o mayor activación de negocios locales, no haya llegado a sus comunidades. En la provincia además no ha habido un mayor desarrollo de un turismo comunitario o de base local, con negocios regentados por miembros de las propias comunidades, como sí ha ocurrido en otras zonas del país, lo cual ha generado un bajo nivel de encadenamiento productivo (Picón y Baltodano, 2005).

La calidad del empleo generado, con acceso solo a empleo poco cualificado, bajos salarios y largas jornadas, se ha convertido también en un elemento crítico. Además, el hecho que una parte de los trabajos de construcción, seguridad, jardinería o limpieza sean asumidos por trabajadores inmigrantes de origen nicaragüense dispuestos a cobrar salarios más bajos ha creado también malestar entre la población local (Navarro, 2013; Navarro, 2014). En este contexto, no se construye un discurso contra el turismo, sino que fundamentalmente se cuestionan los escasos beneficios generados por este modelo concreto y se aspira a un desarrollo turístico más inclusivo (Scheyvens y Biddulph, 2017), en el sentido de una mayor participación de los pobladores de las comunidades en términos tanto de posibilidades de desarrollar negocios propios vinculados al turismo como de mejorar la calidad de los empleos disponibles, o que pueda entroncar con la idea de steady state tourism, en el sentido de la necesidad de fomentar un crecimiento más cualitativo que cuantitativo (Hall, 2009; Hall, 2010).

\section{Conclusiones}

El análisis del caso de Guanacaste y los conflictos socioambientales entre comunidades rurales y desarrollos turístico-residenciales por el agua puede aportar elementos que contribuyen a enriquecer una lectura más compleja de fenómenos descritos en la literatura reciente como overtourism. En contextos rurales-costeros el acceso al agua se ha convertido en uno de los limitantes críticos para el desarrollo turístico-residencial, a la vez que su sobreexplotación puede suponer un claro riesgo para los medios de vida de la población local. De ahí la agudización de conflictos socioambientales vinculados a su control, en una dinámica que revela mecanismos de acumulación por desposesión.

Así las cosas, en estos territorios es posible identificar reacciones de malestar frente al desarrollo turístico similares a las que se producen en destinos urbanos entre sectores de población local frente a los impactos provocados por la masificación turística. Aquí lo que fundamentalmente da lugar a una intensificación de los conflictos es más la percepción de sustracción de recursos naturales básicos que la presencia masiva de turistas. Además, se agudiza en aquellos territorios usados para el desarrollo turístico, pero en los que no se produce propiamente actividades de servicio vinculadas al turismo, por tanto, los posibles beneficios del turismo son aún menores. 
En los movimientos comunitarios más activos en la defensa del agua, que es el sector en el que centramos el interés de este artículo, se identifica un discurso que claramente acusa al desarrollo turístico-residencial de los procesos de despojo. Más allá de los procesos de desplazamiento y reorganización territorial, o bien, la escasa calidad del empleo generado, el agua se convirtió en símbolo de una prosperidad que no les alcanza y de resistencia comunitaria. Con distinta intensidad según los momentos y casos, su posición se articula inicialmente en torno a la demanda de limitación del crecimiento de infraestructuras turísticas, mayor atención a las necesidades más acuciantes de los sectores en situación más vulnerable -antes que dar satisfacción a las demandas de inversionistas y desarrollos turístico-residenciales- $y$, finalmente, la posibilidad de una mayor inclusión en las dinámicas de beneficio generado por el turismo. No se identifica rechazo al turismo, como sí se ha podido observar en movimientos sociales en destinos urbanos en otros contextos, más bien a los procesos de desposesión y desigualdad que ha comportado su desarrollo.

El concepto de overtourism, que empieza a utilizarse recientemente en la literatura académica y en propuestas de política pública, a pesar de tener un recorrido ciertamente corto, tiene la virtud de poner el foco de atención en las expresiones de malestar frente al desarrollo turístico en destinos caracterizados por su éxito en términos de llegadas. Las manifestaciones de desafección o rechazo a este crecimiento aparecen, a la luz de esta perspectiva, como motivos de análisis, en lugar de ser simplemente ignoradas. La asociación entre overtourism y malestar de la población local ante las actividades turísticas, expresado en múltiples formas, desplaza la preocupación analítica y obliga a una reflexión en torno a por qué el crecimiento turístico, en sus múltiples formas, puede dar lugar a insatisfacción entre parte de la población que vive en esos destinos.

Las expresiones de malestar, como ayuda a entender el caso analizado de Guanacaste, pueden dar lugar a más formas que no únicamente el rechazo a esta actividad. Así, los conflictos socioambientales que vinculan agua y turismo en Guanacaste ayudan a ampliar la perspectiva del tipo de reacciones que pueden manifestar comunidades rurales en lugares de una intensa actividad turística. La tensión entre exclusión e inclusión como producto del desarrollo turístico aparece como un marco de análisis que puede ayudar a entender mejor ciertas reacciones y manifestaciones de grupos comunitarios en contextos en los cuales el turismo adquiere una especial relevancia económica.

\section{Agradecimientos}

Este capítulo se ha preparado en el marco del proyecto "Los ODS y el turismo: una estrategia para la educación para el desarrollo", gestionado por Alba Sud con el apoyo financiero de la Agencia Catalana de Cooperación para el Desarrollo (ACCD) 
(convocatoria 2017). Quiero hacer constar mi agradecimiento por la discusión de las ideas presentadas en este artículo y su apoyo en la localización de fuentes de información a: Jordi Gascón (Universidad de Lleida), José Mansilla (Observatorio de Antropología del Conflicto Urbano-OACU), Claudio Milano (Escuela de Turismo y Hostelería de Ostelea-Universidad de Lleida), Ivan Murray (Universidad de las Islas Baleares), Marcela Román (Estado de la Nación), Arturo Silva Lucas (Alba Sud), así como a los revisores anónimos del Anuario de Estudios Centroamericanos.

\section{Bibliografía}

Acuña, G. Flujos migratorios laborales intrarregionales: situación actual, retos y oportunidades en Centroamérica y República Dominicana. Informe de Costa Rica. San José: OIM, OIT, MTSS, CECC SICA, Red de Observatorio del Mercado Laboral, 2011.

Alpízar, F. ¿Democracia ecológica? Las instituciones, la participación política y las contiendas por el agua en Costa Rica (1821-2010) (Tesis doctoral). Madrid: Universidad Complutense de Madrid, 2012.

Ávalos, A. (18 de diciembre de 2008). Estado reclama $\$ 224.000$ a hotel por contaminación de playa Manzanillo. La Nación. Recuperado de https://www.nacion.com/elpais/estado-reclama-224-000-a-hotel-por-contaminacion-de-playa-manzanillo/ GR4TPMMDNFGJPGOVDJKNRCLXGM/story/

Bakker, K. The "Commons" Versus the "Commodity": Alter-globalization, anti-privatization and the human right to water in the global south. Antipode, 39, 3 (2007): 430-455.

Bakker, K. The limits of "neoliberal natures": Debating green neoliberalism. Progress in Human Geography, 34, 6 (2010): 715-735.

Barrantes Reynolds, M. P. "Costa Rica, sin ingredientes artificiales": El rol del Estado en la expansión del turismo residencial en las zonas costeras. Anuario de Estudios Centroamericanos, 39 (2013): 233-261.

Becker, E. Overbooked: The Exploding Business of Travel and Tourism. New York: Simon \& Shuster, 2013.

Bianchi, R. V. (2002). Towards a new political economy of global tourism. Tourism $\mathcal{E}$ Development: Concepts and Issues. R. Sharpley y D. Telfer, eds. Clevedon: Channel View Publications, 265-329.

Bianchi, R. V. (2011). Tourism, capitalism and Marxist political economy. Political Economy of Tourism: A Critical Perspective. J. Mosedale, ed. London and New York: Routledge, 17-37.

Bianchi, R. The Political Economy of tourism development: A critical review. Annals of Tourism Research, 70 (2018): 88-102.

Blanco, E. ¿Testimonios de un despojo? Desarrollo turístico en Guanacaste y sus impactos a nivel social y ambiental, 1990-2016. Revista de Ciencias Sociales, 155 (2017): 13-25.

Blanco, E. ¿Ha valido la pena? Desarrollo turístico en Guanacaste, Costa Rica: resultados a nivel ambiental y social (1990-2016). San José: Universidad de Costa Rica, Vicerrectoría de Investigación, Centro de Investigaciones Históricas de América Central, 2018.

Blàzquez, M., Murray, I. y Antigües, A. La balearizacion global: El capital turístico en la minoración e instrumentación del Estado. Investigaciones turísticas, 2 (2011): 1-28.

Bolaños, C. Conflictos socioambientales por la gestión del agua: el caso de la comunidad de Playa Potrero, Guanacaste (Tesis de licenciatura). San José: Universidad de Costa Rica, 2016. 
Britton, S. G. (1982). The political economy of tourism in the Third World. Annals of tourism research, 9, 3 (1982): 331-358.

Britton, S. G. (1996). Tourism, dependency and development. The Sociology of Tourism. Y. Apostolopoulos, S. Leivadi y A. Yiannakis, eds. London, Routledge, 155-172.

Cabrera, R. Tierra y ganadería en Guanacaste. Cartago: Editorial Tecnológica de Costa Rica, 2007.

Cañada, E. (Coord.). Turismo en Centroamérica: nuevo escenario de conflictividad. Managua: Enlace Editorial, 2010.

Cañada, E. Turismo en Centroamérica. Un diagnóstico para el debate. Managua: Editorial Enlace, 2013.

Cañada, E. (2016). Estalla conflicto por el agua en Playa Panamá, Costa Rica. Entrevista a Gadi Amit. Alba Sud. Recuperado de http://www.albasud.org/blog/es/937/costa-ricaestalla-el-conflicto-por-el-agua-en-playa-panam

Cañada, E. Dispossession, Displacement and Subordination in the Construction of Tourist Areas: Central America as a Conflict Scenario. Norois, 247(2018): 49-61.

Cañada, E. Conflictos por el agua en Guanacaste, Costa Rica. La experiencia de Sardinal de Carrillo. Barcelona: Alba Sud Editorial. Informes en Contraste, 2019.

Cañada, E. y Gascón, J. (2017). Urbanizar el paisaje: turismo residencial, descampesinización, gentrificación rural. Una introducción. Turismo residencial y gentrificación rural. J. Gascón y E. Cañada, eds. El Sauzal (Tenerife): PASOS, Revista de Turismo y Patrimonio Cultural, Colección PASOS Edita, 2017, 5-36.

Cole, S. (2012). A political ecology of water equity and tourism: A case study from Bali. Annals of tourism Research, 39, 2 (2012): 1221-1241.

Cole, S., y Browne, M. (2015). Tourism and water inequity in Bali: A social-ecological systems analysis. Human ecology, 43, 3 (2015): 439-450.

Cole, S., y Ferguson, L. Towards a gendered political economy of water and tourism. Tourism Geographies, 17, 4 (2015): 511-528.

Colomb, C. y Novy, J. (Ed.). Protest and Resistance in the Tourist City. Abingdon: Routledge, 2016.

Cruz, M. F. (11 de octubre de 2016). Gobierno promete planta desalinizadora del pozo de Playa Panamá para diciembre. La Voz de Guanacaste. Recuperado de https://vozdeguanacaste.com/gobierno-promete-planta-desalinizadora-del-pozo-de-playa-panama-paradiciembre/

Edelman, M. The Logic of the Latifundio: The Large Estates of Northwestern Costa Rica since the Late Nineteenth Century. Stanford: Stanford University Press, 1992.

Estado de la Nación. Estado de la Nación 2017. San José: Programa del Estado de la Nación, 2017.

Fernández, Ó. Papagayo, Sardinal y la gobernanza en Costa Rica: dos intervenciones del Estado y sus distintas consecuencias. Reflexiones. Revista Facultad Ciencias Sociales Universidad de Costa Rica, 88, 2, 2009, 9-16.

Girardi Stellin, V. Carta Pastoral de Monseñor Victorino Girardi "Discípulos, tras las huellas de Cristo, promoviendo la vida en Él, al Norte de Costa Rica". Liberia: Diócesos de TilaránLiberia, 2009.

Gordon, B., Sarmiento, F., Russo, R. y Jones, J. Sustainability Education in Practice: Appropriation of Rurality by the Globalized Migrants of Costa Rica. Journal of Sustainability Education, 1 (2010). Recuperado de https://100noticias.com.ni/nacionales/94813-crisis-nicaraguaprotesta-represion

Hall, C. M. Degrowing tourism: Décroissance, sustainable consumption and steady-state tourism. Anatolia, 20, 1 (2009): 46-61. 
Hall, C. M. Changing paradigms and global change: From sustainable to steady-state tourism. Tourism Recreation Research, 35, 2 (2010): 131-143.

Harvey, D. The new imperialism. Oxford: Oxford University Press, 2003.

Harvey, D. Seventeen Contradictions and the End of Capitalism. Oxford: Oxford University Press, 2014.

Hernández, A. y Picón, C. En la frontera del conflicto socio-ambiental: el modo de vida rural y el desarrollo del turismo de sol y playa en Guanacaste, Costa Rica. Introducción. Ambientales. Revista Semestral de Escuelas de Ciencias Ambientales, 42, 2 (2011): 31-44.

Hernández, A. y Picón, J. C. Huella hídrica en tierras secas: el caso del turismo de sol y playa en Guanacaste (Costa Rica). Revista de Ciencias Ambientales, 45, 1 (2013): 41-50.

Hof, A. y Blázquez-Salom, M. The Linkages between Real Estate Tourism and Urban Sprawl in Majorca (Balearic Islands, Spain). Land, 2, (2013): 252-277.

Honey, M., Vargas, E. y Durham, W. H. Impacto del turismo relacionado con el desarrollo en la Costa Pacifica de Costa Rica. Washington, D. C.: Center for Responsible Travel (CREST), 2010.

Irazábal, C. Coastal Urban Planning in The 'Green Republic': Tourism Development and the Nature-Infrastructure Paradox in Costa Rica. International Journal of Urban and Regional Research, 42, 5 (2018): 882-913.

Janoschka, M. The contested spaces of lifestyle mobilities: Regime analysis as a tool to study political claims in Latin American retirement destinations. Erde, 140, 3 (2009): 251-274.

Janoschka, M. (2011). Imaginarios del turismo residencial en Costa Rica. Negociaciones de pertenencia y apropiación simbólica de espacios y lugares: una relación conflictiva. Construir una nueva vida: los espacios del turismo y la migración residencial. T. Mazón, R. Huete y A. Mantecón, eds. Santander: Milrazones, 81-102.

Kuzdas, C. Unpacking water conflict in Guanacaste, Costa Rica. Canberra: Global Water Forum, Discussion Paper 1242, 2012.

Lizano, R. Futuro del desarrollo turístico en los litorales de Costa Rica. Ambientales. Revista Semestral de Escuelas de Ciencias Ambientales, 42 (2011): 7-17.

Martínez-Alier, J. Los conflictos ecológico-distributivos y los indicadores de sustentabilidad. Polis, 13 (2006). Recuperado de https://journals.openedition.org/polis/5359

Milano, C. Overtourism, malestar social y turismofobia. Un debate controvertido. PASOS. Revista de Turismo y Patrimonio Cultural, 18, 3 (2018): 551-564.

Milano, C., Cheer, J. M. y Novelli, M. (18 de julio de 2018). Over tourism: a growing global problem. The Conversation. Recuperado de https://theconversation.com/overtourisma-growing-global-problem-100029

Milano, C., Cheer, J. y Novelli, M. (Eds.). Overtourism: Excesses, discontents and measures in travel and tourism. Wallingford: CABI, 2019.

Milano, C. y Mansilla, K. (Eds.). Ciudad de vaciones. Conflictos urbanos en espacios turísticos. Barcelona: Pol·len Edicions, 2018.

Morales, A. (Coord.). Migraciones y derechos laborales en Centroamérica: características de las personas migrantes y de los mercados de trabajo. San José: FLACSO, 2012.

Navarro, S. (2013). Turismo e inmigración en Playa Matapalo, Sardinal, Costa Rica. Resistencias comunitarias y laborales. Anuario de Estudios Centroamericanos, 39 (2013): 263-287.

Navarro, S. Geopolítica en una "periferia del placer". Colonialidad turística en Costa Rica. Revista Ciencias Sociales, 145 (2014): 45-60.

Navas, G. El agua fluye hacia el turista. Letras Verdes. Revista Latinoamericana de Estudios Socioambientales, 18 (2015): 27-47. 
Navas, G. y Cuvi, N. Análisis de un conflicto socioambiental por agua y turismo en Sardinal, Costa Rica. Revista Ciencias Sociales, 150, IV (2015): 109-124.

Van Noorloos, F. Whose place in the sun? Residential tourism and its implications for equitable and sustainable development in Guanacaste, Costa Rica. Utrecht: Eburon, 2012.

Van Noorloos, F. Residential tourism and multiple mobilities: Local citizenship and community fragmentation in Costa Rica. Sustainability (Switzerland), 5, 2 (2013): 570-589.

Van Noorloos, F. y Steel, G. Lifestyle migration and socio-spatial segregation in the urban(izing) landscapes of Cuenca (Ecuador) and Guanacaste (Costa Rica). Habitat International, 54 (2016): 50-57.

Pera, J. L. Tamagringo: citizenship and community change in Tamarindo, Costa Rica. Oregon: Univerty of Oregon-Department of Geography, Master of Arts, 2008.

Picón, J. C. y Baltodano, V. Capacidad competitiva de las microempresas de hospedaje. El caso de las comunidades cercanas al proyecto turístico Papagayo, Guanacaste, Costa Rica. InterSedes: Revista de Las Sedes Regionales Universidad de Costa Rica, 6, 10-11 (2005). Recuperado de https://revistas.ucr.ac.cr/index.php/intersedes/article/view/784

Picón, J. C. y Baltodano, V. Planificación turística en zonas costeras de Costa Rica. Algunas referencias a Playa Tamarindo (Santa Cruz, Guanacaste). InterSedes: Revista de Las Sedes Regionales Universidad de Costa Rica, 7, 13 (2006): 149-170.

Picón, J. C., Hernández, A. y Bravo, J. (2014). Cambio climático, transformaciones en la línea costera y turismo de sol y playa en la costa pacífico-norte costarricense. Ambientico, 246 (2014): 16-21.

Quirós, B. (27 de diciembre de 2016). Denuncian agua contaminada por salinización en Guanacaste. La Prensa Libre. Recuperado de www.laprensalibre.cr/Noticias/detalle/ 98327/denuncian-agua-contaminada-por-salinizacion-en-guanacaste-

Ramírez, A. (2008). Conflictos socioambientales y recursos hídricos en Guanacaste; una descripción desde el cambio en el estilo de desarrollo (1997-2006). Anuario de Estudios Centroamericanos, 33-34 (2008): 359-385.

Retana, K. (7 de febrero de 2008). Salud cierra hotel Allegro Papagayo. La República. Recuperado de https://www.larepublica.net/noticia/salud_cierra_hotel_allegro_papagayo

Román, I. Diversidad de destinos y desafíos del turismo en Costa Rica: los casos de Tamarindo y La Fortuna. En Decimotercer Informe Estado de la Nación en Desarrollo Humano Sostenible (pp. 193-226). San José: Programa Estado de la Nación, 2006.

Román, M. Desarrollo turístico e inmobiliario costero y preocupaciones ambientales. En Decimotercer Informe sobre el Estado de la Nación en Desarrollo Humano Sostenible (pp. 1-19). San José: Programa Estado de la Nación, 2007.

Román, M. Costa Rica: Dinámica del mercado inmobiliario en la costa pacífica. San José: Center on Ecotourism and Sustainable Development, 2009.

Saarinen, J. Traditions of sustainability in tourism studies. Annals of Tourism Research, 33, 4 (2006): 1121-1140.

Sancho, M. (20 de octubre de 2018). Marbella: el pueblo en el que un desarrollador se otorga a sí mismo permisos de agua. Crhoy. Recuperado de https://www.crhoy.com/ambiente/marbella-el-pueblo-en-el-que-un-desarrollador-se-otorga-si-mismo-permisos-deagua/

Scheyvens, R. y Biddulph, R. Inclusive tourism development. Tourism Geographies, 20, 4 (2018): 589-609. 
Scoot, J. C. Weapons of the Weak. Everyday Forms of Peasant Resistance. New Haven: Yale University Press, 1985.

Silva, A. El movimiento social por el agua en Sardinal de Guanacaste (Tesis de licenciatura). San José: Universidad de Costa Rica, 2016.

Silva, A. (5 de diciembre de 2018). Lorena (Guanacaste): Agua, territorio y espacios de representación. Alba Sud. Recuperado de http://www.albasud.org/noticia/es/1076/lorenaguanacaste-agua-territorio-y-espacios-de-representaci-n

Silva, A. (2019). Conflictividad hídrica en Guanacaste como consecuencia del desarrollo turístico: el caso de la comunidad de Sardinal. Acciones Colectivas y Contiendas Políticas por el Agua en Costa Rica. F. Alpízar, ed. San José: PNUD-CIEP, 2019, en prensa.

Silva, A. y Morera, C. El proceso de gentrificación en el actual modelo turístico de Costa Rica: Claves para su interpretación (comunicación). En XI Congreso Internacional sobre turismo rural y desarrollo sustentable, CITURDES, Nicola, Costa Rica, 14-16 de noviembre de 2018.

UNWTO et al. 'Overtourism'?-Understanding and Managing Urban Tourism Growth beyond Perceptions. Madrid: UNWTO, 2018.

UNWTO et al. 'Overtourism'?-Understanding and Managing Urban Tourism Growth beyond Perceptions. Volumen 2: Cases studies. Madrid: UNWTO, 2019.

Ernest Cañada. Español. Coordinador del Centro de Investigación Alba Sud y docente del Campus del Turismo, Hotelería y Gastronomía de la Universidad de Barcelona (CETT-UB). Candidato a Doctor en Historia y Geografía por la Universidad de las Islas Baleares (UIB). Posgrado en Demografía por el Centro de Estudios Demográficos de la Universidad Autónoma de Barcelona (UAB).

Contacto: ernest@albasud.org

ORCID: 0000-0001-6086-1644 